\title{
Variation of proton fluxes of galactic cosmic rays during 2012-2020 according to data from the Russian spacecraft in geostationary orbit
}

Evgeny A. Bondarev, ${ }^{a, *}$ Alexander E. Koziukov, ${ }^{a}$ Grigory A. Protopopov, ${ }^{a}$ Pavel A. Chubunov, ${ }^{a}$ Andrey Y. Repin, ${ }^{b}$ Valentina I. Denisova ${ }^{b}$ and Alexey V. Tsurgaev $^{b}$

${ }^{a}$ Institute of Space Device Engineering,

Aviamotornaya st. 55, Moscow, Russia

${ }^{b}$ Fedorov Institute of Applied Geophysics,

Rostokinskaya st. 9, Moscow, Russia

E-mail: evgeniy.bondarev3@gmail.com, conf@niikp.org

In this paper, we study the effect of solar modulation over the past decade, and also conduct a comparative analysis with the data provided by the GOES spacecraft. The measurements were carried out using the Russian spacecraft on the geostationary orbit. The detector has 5 channels for detecting protons with energies $\mathrm{E} 3.5 \mathrm{MeV}, \mathrm{E} 15 \mathrm{MeV}$ and also with energies $\mathrm{E}=13.7-23$ $\mathrm{MeV}, \mathrm{E}=23-42 \mathrm{MeV}, \mathrm{E}=42-112 \mathrm{MeV}$.

$37^{\text {th }}$ International Cosmic Ray Conference (ICRC 2021)

July 12th-23rd, 2021

Online - Berlin, Germany

\footnotetext{
*Presenter
} 


\section{Introduction}

The Russian spacecraft works on the GEO for most of the 24 solar activity cycle and this is another tool for monitoring space weather. We have made a brief overview of the measurements of the Russian spacecraft on the GSO. In the course of the work, a comparison was also made with the goes data. To compare the data, time profiles of integrated flows from GOES and the Russian spacecraft were constructed. Energy spectra are also constructed.

\section{On-board measurements used, proton spectra and time profiles}

We used two datasets from GOES [1] and Russian GEO [2] spacecrafts:

- GOES daily data: protons with energies $E \geq 1 \mathrm{MeV}, E \geq 10 \mathrm{MeV}, E \geq 100 \mathrm{MeV}$;

- Geostationary orbit spacecraft on-board measurements include fluxes of protons with different energy ranges: from $13.7 \mathrm{MeV}$ to $23 \mathrm{MeV}$; from $23 \mathrm{MeV}$ to $42 \mathrm{MeV}$; from $42 \mathrm{MeV}$ to 112 $\mathrm{MeV}$;

On the Figure 1 are the spectra of protons on the GEO according to the GOES and Russian spacecraft data. Summarized integral flux on the plots is summed up for each year. As can be seen from the plots, there is a good agreement between the spacecraft until 2015, but after that there are significant differences. The reason for their occurrence is not clear yet.

On the Figure 2 is a time series of changes in proton fluxes on the GEO according to data from the Russian spacecraft in GEO (upper fig.) and from GOES (lower). In this case, we are talking about variations of proton fluxes in the heliosphere, where contribution to the greatest peaks is obviously made by solar flares, as indicated by peaks in the regions of 2012 and 2017, when the most powerful flares of the 24 th cycle occurred. There is also an agreement between similar energy channels: $13.7 \mathrm{MeV}$ for the Russian spacecraft and $10 \mathrm{MeV}$ for GOES 

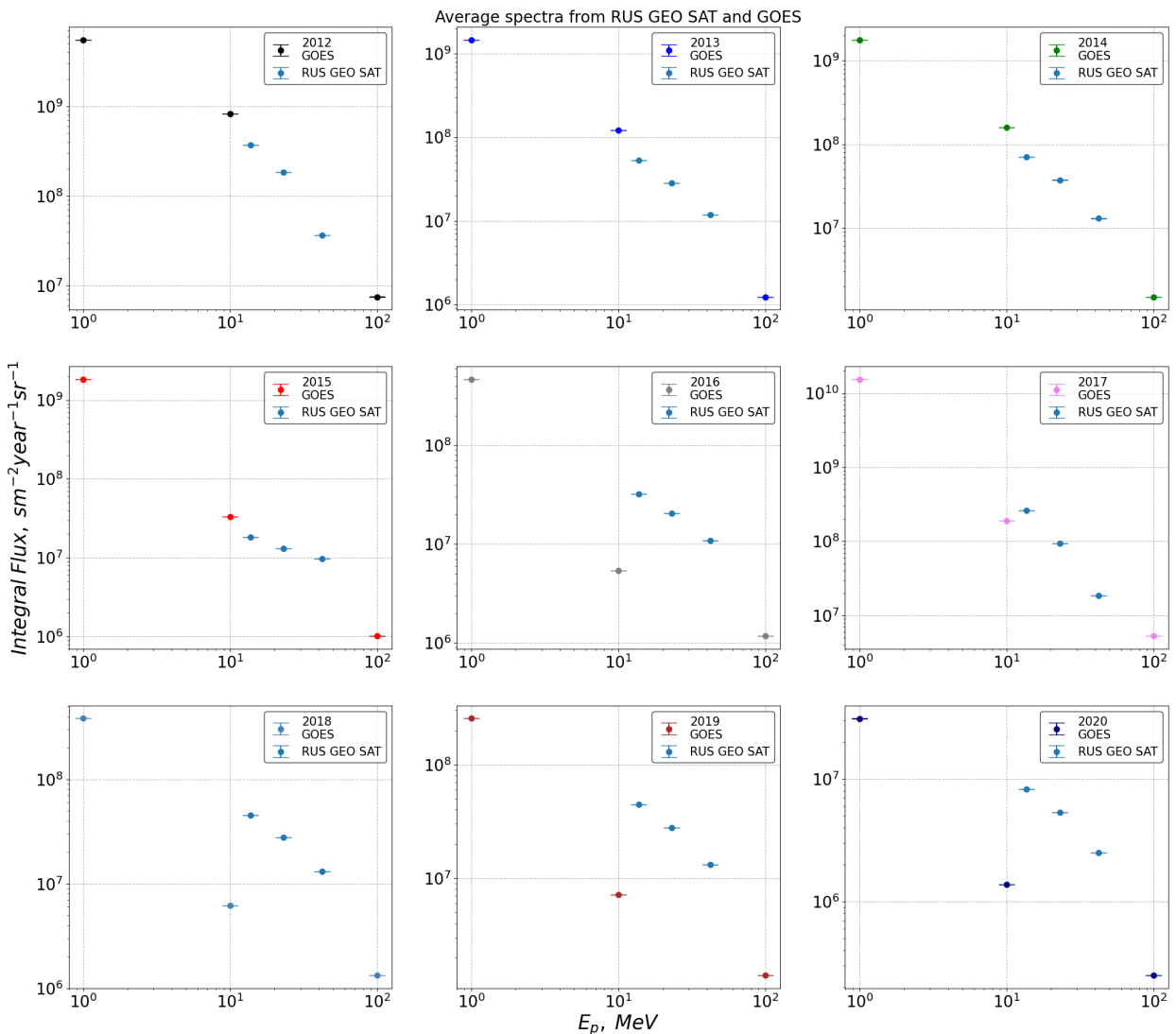

Figure 1: Proton spectra in GEO 

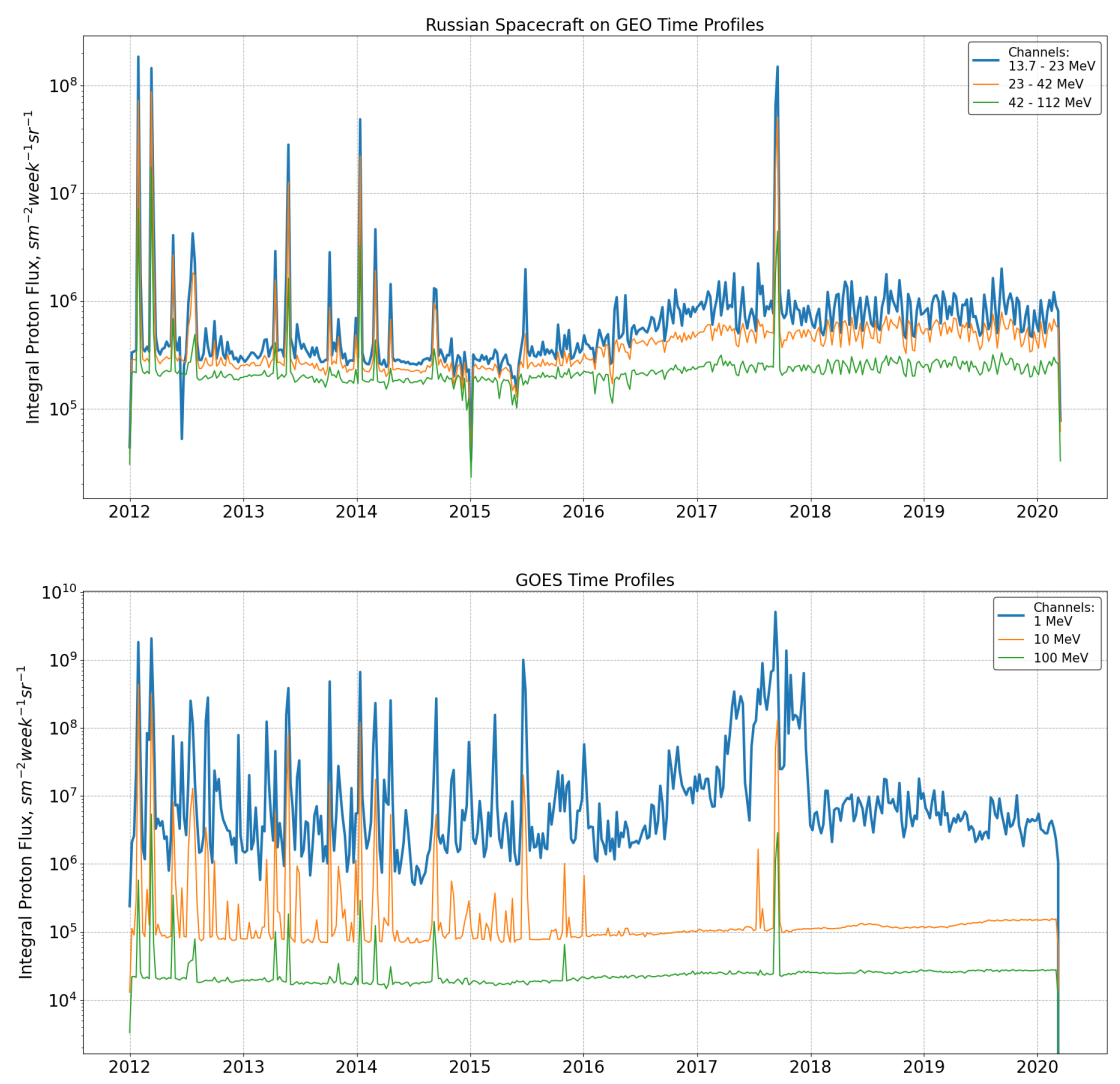

Figure 2: Time profiles for the GOES and Russian GEO spacecraft

\section{Conclusions}

The energy spectra of the galactic protons in the GEO, measured by GOES and the Russian spacecraft, are in good agreement until 2015, after that there is a noticeable discrepancy. In turn, the time profiles of proton fluxes have a good agreement throughout the entire period under consideration. In addition, the key moments with powerful flashes are clearly visible on the time series. The review done largely illustrates the qualitative compliance of the GOES data with similar data of the Russian spacecraft in GSO and indicates the possibility of effective monitoring of space weather using the RF spacecraft in GEO.

\section{References}

[1] http://wWw.ngdc.noaa.gov/stp/satellite/goes/dataaccess.html 
[2] V. S. Anashin, G. A. Protopopov, and Y. A. Milovanov, "Monitoring of space radiation in Russian federal space agency", 12th European Conference on Radiation and Its Effects on Components and Systems, Sep. 2011. 